\title{
Estimativa do fluxo de nitrogênio na universidade federal da Sergipe, Brasil
}

Este artigo discute o problema das alterações no ciclo de nitrogênio e apresenta a identificação e estimativa dos fluxos anuais de nitrogênio reativo no Campus São Cristóvão da Universidade Federal de Sergipe. Através deste estudo, foi possível analisar muitos aspectos ambientais intrinsecamente ligadas às atividades do campus, tais como o consumo de energia, transporte, práticas de jardinagem, consumo de alimentos, geração de efluentes e outros aspectos responsáveis por diversos impactos ambientais no campus. Com base na identificação das atividades bem como na estimativa dos cálculos de emissão de $\mathrm{N}$ do referido campus, verificou-se que o campus é responsável por gerar mais de $40.000 \mathrm{~kg} \mathrm{~N}$ ano-1, sendo que a maior entrada de $\mathrm{N}$ é responsável por cerca de $30 \%$ do total de emissõe e é referente aos alimentos que entram no campus e são consumidos nas lanchonetes e restaurantes. Tendo em vista as três maiores emissões estimadas nesse campus, foram sugeridas neste trabalho algumas alternativas de natureza preventivas pautadas em conceitos e métodos de tecnologias limpas e ecologia industrial voltadas a redução dessas emissões. Os resultados apresentados neste estudo permitem a identificação dos impactos ambientais relacionados com a geração do nitrogênio reativo e que pode funcionar como um instrumento de orientação para a adoção de políticas ambientais e educacionais para o campus.

Palavras-chave: Campus ecológico; Nitrogênio no campus; Fluxo de nitrogênio; Campus sustentáveis.

\section{Nitrogen flow estimation at the federal university of Sergipe, Brazil}

This paper discusses the problem of changes in the nitrogen cycle and presents the identification and estimation of annual reactive nitrogen fluxes at the São Cristóvão Campus of the Federal University of Sergipe. Through this study, it was possible to analyze many environmental aspects intrinsically related to campus activities, such as energy consumption, transportation, gardening practices, food consumption, effluent generation and other aspects responsible for various environmental impacts on campus. Based on the identification of the activities as well as on the estimation of the $\mathrm{N}$-emission calculations of said campus, it was verified that the campus is responsible for generating more than $40,000 \mathrm{~kg} \mathrm{~N}$ year-1, with the largest entrance of $\mathrm{N}$ being responsible for about of the total emissions and refers to foods that enter the campus and are consumed in snack bars and restaurants. Considering the three largest emissions estimated on this campus, some preventive alternatives based on concepts and methods of clean technologies and industrial ecology aimed at reducing these emissions were suggested in this paper. The results presented in this study allow the identification of the environmental impacts related to the generation of reactive nitrogen and can act as a guiding instrument for the adoption of environmental and educational policies for the campus.

Keywords: Greening campus; Nitrogen Campus; Nitrogen flows; Campus sustainability.

\section{Topic: Engenharia Ambiental}

Reviewed anonymously in the process of blind peer.
Received: 10/04/2018

Approved: 24/05/2018
Rodrigo Gallotti Lima (10)

Universidade Federal de Sergipe, Brasil http://lattes.cnpq.br/6838783610597692 http://orcid.org/0000-0002-0786-7358 florafertil@yahoo.com.br

Anita Maria Lima (iD)

Universidade Federal de Sergipe, Brasil http://lattes.cnpq.br/9805293444879480

http://orcid.org/0000-0002-0851-2815

anita.lima@gmail.com

\section{Laura Jane Gomes (iD)}

Universidade Federal de Sergipe, Brasil http://lattes.cnpq.br/2534479131206357

http://orcid.org/0000-0003-1526-7456

laurabuturi@gmail.com
Sergio Luis Martins dos Santos

Universidade Federal de Sergipe, Brasil

http://lattes.cnpq.br/7195752704659454

srgsantos0412@gmail.com

\section{Referencing this:}

LIMA, R. G.; LIMA, A. M.; GOMES, L. J.; SANTOS, S. L. M.. Estimativa do fluxo de nitrogênio na universidade federal da Sergipe, Brasil. Revista Ibero Americana de Ciências Ambientais, v.9, n.4, p.224-234, 2018. DOI: $\underline{\text { http://doi.org/10.6008/CBPC2179-6858.2018.004.0019 }}$ 


\section{INTRODUÇÃO}

$\mathrm{O}$ ar atmosférico tem cerca de $78 \%$ de nitrogênio molecular $\left(\mathrm{N}_{2}\right)$, um gás inerte que nem a maioria das plantas podem usá-lo como nutriente. No entanto, em 1908, Fritz Haber criou um método para transformar $\mathrm{N}_{2}$ em amônia, tendo em vista a possibilidade de incrementar a produção de fertilizantes sintéticos. Em 1913, o cientista Carl Bosh, desenvolveu o método criado por Fritz expandindo-o para ser executado em escala industrial. Isso o levou ao Prêmio Nobel em 1931. A invenção denominada 'Processo Haber-Bosch' foi de fundamental importância para humanidade, pois, foi capaz de elevar significativamente o patamar da produção bélica e agrícola a nível mundial (ERISMAN et al., 2008; FOWLER et al., 2013; GALLOWAY et al., 2013; 2014; ERISMAN, 2015). Mundialmente, cerca de 75\% da produção de Nitrogênio reativo $\left(N_{r}\right)$ antropogênico decorre de fixação industrial de $N$ e $25 \%$ a partir de combustíveis fósseis e da queima de biomassa, sob a forma de $\mathrm{NO}_{x}$ (GALLOWAY et al., 2013; FOWLER et al., 2013).

Atualmente, só a produção de $\mathrm{N}_{\mathrm{r}}$ voltada para agricultura é mais do que o dobro da quantidade natural pré-industrial produzida nos ecossistemas terrestres (FOWLER et al., 2013; ERISMAN, 2015). Diante disso, surgiram diversas consequências ambientais catastróficas por conta da alteração do Ciclo do $\mathrm{N}, \mathrm{a}$ exemplo: surgimento de zonas costeiras mortas e mortandade de peixes devido à eutrofização grave ou hipóxia resultante do escoamento nitrato e da lixiviação em sistemas fluviais; Redução da biodiversidade em mananciais devido à eutrofização e acidificação; Poluição da água subterrânea por nitratos; Poluição de água doce devido à eutrofização e acidificação; Impactos na saúde humana devido a formação de aerossóis e de $\mathrm{O}_{3}$ troposférico causando diversas doenças; Redução das colheitas, das florestas e da produtividade de pastagem devido à deposição de nitrogênio e excesso de fertilização, bem como a exposição ao ozônio troposférico; Aumento das mudanças climáticas da Terra e aumento da depleção da camada de ozônio estratosférico etc (GALLOWAY, 2008; ROCKSTROM et al., 2009; GALLOWAY et al., 2003; 2004; 2014; ERISMAN et al., 2013; 2015; FOWLER et al., 2013; 2015; UNEP, 2014).

Um século após a invenção do processo Haber-Bosch o planeta passou a emitir cerca de 210.000 .000 de toneladas de $\mathrm{N}_{\mathrm{r}}$. Para se ter ideia, a fixação de nitrogênio através desse processo em 2010 (120.000.000 de toneladas $\mathrm{N}_{r}$ ano-1) representou o dobro do total de todas as fontes terrestres naturais de $\mathrm{N}_{r}(63.000 .000$ toneladas $\mathrm{N}_{\mathrm{r}}$ ano-1$^{-1}$ ) (FOWNLER et al., 2013, 2015). O fator que agrava ainda mais essa problemática se dá pelo fato de apenas um único átomo de $\mathrm{N}_{\mathrm{r}}$ contribuir para os mais diversos efeitos negativos de natureza socioeconômica e ambientais. De acordo com a UNEP (2014) o nitrogênio move-se através do ambiente e o mesmo átomo de $\mathrm{N}$ pode contribuir para múltiplos impactos negativos, seja no ar, em terra, em sistemas de água doce e marinhos e também sobre a saúde humana. A esta sequência contínua durante um longo período denomina-se 'Cascata de N', tema exaustivamente discutido há mais de uma década por cientistas como Galloway e Erisman (ERISMAN et al., 2007, 2008, 2011, 2013; GALLOWAY, 1998, 2003; GALLOWAY et al., $2003 ; 2004 ; 2008 ; 2013 ; 2014)$.

Desde algum tempo, Rockstrom et al. (2009) e Steffen et al. (2015), vem chamando a atenção da comunidade científica, divulgando alertas relativo às ações humanas que já ultrapassaram a nível mundial os 
limites seguros no tocante ao ciclo de nitrogênio, dentre outras questões ambientais. Segundo os autores, os limites são compostos por processos imbuídos em sistemas naturais que influenciam na capacidade de resiliência do Planeta Terra. Após a identificação dos processos, os autores propuseram fronteiras seguras para cada um, o que, se ultrapassadas, promovem condições ambientais adversas que condicionam risco de vida de muitas espécies, incluindo os seres humanos. Depois de analisar cada sistema separadamente, os autores concluíram que três deles já ultrapassaram o limite: Perda de Biodiversidade, Ciclo do Nitrogênio e Mudanças Climáticas. No caso do Ciclo do $\mathrm{N}$, em uma escala global, a quantidade de $\mathrm{N}_{2}$ convertidos em $\mathrm{N}_{\mathrm{r}}$ das atividades humanas é muito elevado e tem alterado perigosamente o biogeociclo do nitrogênio.

Questões ambientais têm despertado em muitas instituições a adoção de ações, políticas, planos diretores, dentre outras posturas de atuação voltadas a promoção da sustentabilidade nestas instituições. Diversos são os focos que tem mobilizado as Instituições de Ensino Superior (IES), a exemplo dos ciclos biogeoquímicos, mudanças climáticas, uso racional de recursos naturais dentre outras, para a realização de iniciativas mais sustentáveis (UMACS, 2013). Relacionado a isso, duas instituições direcionaram seus esforços para a questão do ciclo N, sendo uma abordada na pesquisa Savanick et al. (2007), que teve como objetivo quantificar as entradas e saídas anuais de nitrogênio reativo do Campus Twin Cities, Universidade de Minnesota. A segunda foi a Universidade de Virginia, contemplada na pesquisa Leach, et al., (2013) considerado o primeiro modelo em nível de universidade que estimou a pegada de nitrogênio da Universidade de Virginia (UVA), tanto a atual como a projetada para 2025. Este modelo também é usado para testar cenários sobre as formas mais eficazes para reduzir a pegada de nitrogênio $(\mathrm{N})$ da universidade. No Brasil, até os dias atuais não há nenhum registro sobre as iniciativas focadas em ciclo N. Neste sentido, o objetivo deste trabalho foi identificar e estimar os fluxos de $\mathrm{N}_{r}$, gerados pelas demandas das atividades existentes no Campus São Cristóvão de Universidade Federal de Sergipe (UFS).

\section{METODOLOGIA}

Esta pesquisa tem foco apenas nos fluxos de $\mathrm{N}_{\mathrm{r}}$ gerados pelas demandas do campus, uma vez que o problema relacionado com o ciclo do nitrogênio é do aumento de $N_{r}$ na atmosfera, que é resultante das ações antrópicas. Para estimativa das emissões considerou-se um ano escolar com 200 dias, por representar mais significativamente as contribuições do campus no ciclo de nitrogênio. Para fins de padronização das emissões, durante os cálculos, todas emissões em $\mathrm{g}$ de $\mathrm{NO}_{x}$ foram convertidas para serem expressas em $\mathrm{kg}$ de $\mathrm{N}$ ano ${ }^{-1}$, logo, durante nos cálculos foi utilizado tanto o fator de transformação de 0,001 (referente a conversão de $\mathrm{g} \rightarrow \mathrm{kg}$ ) como o fator de conversão de $\mathrm{NO}_{x}$ em $\mathrm{N}$, pois, para a massa de $\mathrm{N}$ presente em $\mathrm{NO}_{x}$ foi adotado, para todos os cálculos, a proporção utilizada para $\mathrm{NO}_{2}$ (massa $\mathrm{N} / \mathrm{NO}_{2} \rightarrow$ massa $=14 / 46$ ). Já para calcular a massa de $\mathrm{N}$ presente no $\mathrm{NH}_{\mathrm{x}}$ depositado foi utilizada a proporção de $\mathrm{N}$ presente em $\mathrm{NH}_{3}$ e $\mathrm{NH}_{4}$ $((14 / 17+14 / 18) / 2)$ 


\section{Campus São Cristóvão - UFS}

De acordo com os dados fornecidos pela prefeitura do Campus São Cristóvão da UFS, o mesmo possui uma área total de 1.542.807 $\mathrm{m}^{2}$ (154,3 ha), sendo 443.969,67 $\mathrm{m}^{2}$ (44,4 ha) de área urbana e 1.098.837,83 $\mathrm{m}^{2}$ (109,9 ha) de área verde, caracterizada pela presença da Mata Atlântica. O Campus é composto por vários prédios, onde funcionam a Reitoria, a Prefeitura do Campus, o Setor Esportivo, os Centros Acadêmicos (CCBS, CCET, CCSA e CECH), a Biblioteca Central - BICEN, o Restaurante Universitário - RESUN, o Núcleo de Tecnologia da Informação - NTI, o Arquivo Central, o Centro Editorial e Audiovisual - CEAV, Colégio de Aplicação - CODAP, onde frequenta uma população acadêmica composta de servidores públicos, terceirizados e alunos que totaliza cerca de 20.000 pessoas.

Para estimativa das emissões foram considerados dados dos anos de 2015 e 2016, sendo que, em 2015 o campus em estudo teve apenas os 98 dias ativos, já em 2016 foram 184 dias ativos. Os dias de greve não foram contabilizados nessa pesquisa por não representar fidedignamente a contribuição do campus no tocante a sua geração de $\mathrm{N}$ reativo. Para realização desta pesquisa foram realizadas visitas técnicas ao Campus São Cristóvão a fim de serem obtidos os dados demandados para os cálculos das emissões de $\mathrm{N}$ pelo referido campus.

\section{Fontes de dados para estimativa das emissões}

No que se refere a estimativa das emissões de $N_{r}$ no referido campus, a pesquisa baseou-se no modelo de quantificação de cálculo de $\mathrm{N}_{\mathrm{r}}$ desenvolvido por Savanick et al. (2007), que se propôs a estimar as referidas emissões decorrentes de atividades demandadas pelo campus. Os dados foram obtidos em diversos setores administrativos do campus. As fontes dos dados são detalhadas no Quadro 1.

Quadro 1: Fontes dos dados utilizados para pesquisa e modelo matemático utilizado na estimativa.

\begin{tabular}{|c|c|}
\hline Fontes & UFS \\
\hline Energia & $\begin{array}{l}\text { Considerou-se as emissões de } \mathrm{N} \text { provenientes de geração da energia elétrica que foi consumida no campus } \\
\text { durante o período referente ao } 2015 \text {. Esse dado foi obtido pelas contas de energia mediante o setor de gestão } \\
\text { ambiental do campus. Já o fator de emissão de } \mathrm{NO}_{x} \text { foi obtido pelo ECOINVENT. }\end{array}$ \\
\hline $\begin{array}{l}\text { Transportes } \\
\text { terrestres e } \\
\text { aéreo }\end{array}$ & $\begin{array}{l}\text { Meios de transporte utilizados pela comunidade acadêmica (carros, ônibus e aeronaves) - Em relação às } \\
\text { emissões de transporte, foi considerado o } \mathrm{N} \text { emitido pelos veículos oficiais da universidade, veículos } \\
\text { particulares e ônibus que transportam a comunidade acadêmica. Além disso, foram contempladas as emissões } \\
\text { geradas a partir de viagens aéreas. A frota de veículos oficiais bem como os combustíveis utilizados por eles } \\
\text { foi obtida no setor de transportes do campus. A população acadêmica total foi informada pelo setor } \\
\text { administrativo do campus. A média diária de carros particulares que transitam no campus, bem como a } \\
\text { distância média percorrida dos usuários do campus foram obtidos através do setor de gestão ambiental do } \\
\text { campus. A quantidade de usuários de ônibus que se deslocam para o campus bem como a distância percorrida } \\
\text { por eles por dia, fora obtida através do setor de gestão ambiental do campus. O fator de emissão de } \mathrm{NO}_{x} \text { dos } \\
\text { carros, vans e ônibus foi obtido por MMA (2013). } \\
\text { Para o transporte aéreo foi computada a quilometragem total dos voos realizados referente ao ano. Os } \\
\text { destinos percorridos nos voos realizados por professores e alunos foram obtidos na pró-reitoria de pós- } \\
\text { graduação da universidade. O fator de emissão de NO } \mathrm{O}_{x} \text { referente ao querosene de aviação, bem como a } \\
\text { densidade desse combustivel foi obtido a partir da ANAC (2014). O tipo e os dados da aeronave mais utilizada } \\
\text { nos voos foram obtidos a partir da CONDOR. } \\
\text { Cabe ressaltar que neste trabalho foi considerada a quantidade de nitrogênio reativo emitido por pessoa em } \\
\text { cada meio de transporte estudado. Para isso, em cada veículo, foi identificada a lotação máxima do mesmo. } \\
\text { Em seguida o respectivo fator de emissão utilizado nos cálculos foi dividido pela lotação máxima em cada caso. } \\
\text { Neste trabalho as lotações máximas consideradas foram: veículos leves - } 5 \text { pessoas; vans - } 12 \text { pessoas; ônibus } \\
\text { - } 46 \text { pessoas e avião - } 220 \text { pessoas. Já para o computo do } \mathrm{N}_{\mathrm{r}} \text { emitido dos carros oficiais, não foi necessário } \\
\text { considerar a lotação desses veículos, pois, o setor de transporte da universidade informou dados de km } \\
\text { percorrido por dia referente a todos os seus veículos. }\end{array}$ \\
\hline
\end{tabular}




\begin{tabular}{|l|l|}
\hline Alimentos & $\begin{array}{l}\text { A relação de alimentos consumidos no campus foi fornecida pelos proprietários e/ou os setores } \\
\text { administrativos de restaurantes. O teor de proteína de cada tipo de alimento foi obtido a partir da Tabela } \\
\text { Brasileira de Alimentos (UNICAMP, 2011). A percentagem de proteína em N foi obtida a partir de Esminger } \\
\text { (1991) citado por Savanick et al. (2007). }\end{array}$ \\
\hline $\begin{array}{l}\text { Resíduos de } \\
\text { alimentos }\end{array}$ & $\begin{array}{l}\text { Os resíduos de alimentos desperdiçados tanto na preparação quanto nas sobras das refeições servidas em } \\
\text { restaurantes foram fornecidos pelo setor administrativo de cada restaurante do campus. Com essa quantidade } \\
\text { e com o percentual de N (obtida via Tabela Brasileira de Alimentos, UNICAMP, 2011) de cada alimento } \\
\text { discriminado, obteve-se o total da emissão referente aos resíduos dos restaurantes em de kg N ano-1 }\end{array}$ \\
\hline $\begin{array}{l}\text { Esgoto } \\
\text { alimentos }\end{array}$ & $\begin{array}{l}\text { Para o cálculo do esgoto gerado, levou-se em consideração a quantidade de } \mathrm{N} \text { (obtida via Tabela Brasileira de } \\
\text { Alimentos, UNICAMP, 2011) no alimento ingerido e depois multiplicado por 90 \%, pois 90\% dos alimentos } \\
\text { ingeridos viram esgoto, segundo Baker et al., 2001. }\end{array}$ \\
\hline FBN & $\begin{array}{l}\text { A FBN da Mata Atlântica foi obtida a partir do mapeamento de Filoso et al. (2006); A área verde do campus foi } \\
\text { obtida nos projetos de arquitetura fornecidos no setor de planejamento da prefeitura do campus. }\end{array}$ \\
\hline $\begin{array}{l}\text { Deposição } \\
\text { Atmosférica de } \\
\mathbf{N}\end{array}$ & $\begin{array}{l}\text { A deposição atmosférica do nitrogênio foi obtida pelos mapas de deposição modelados de } \mathrm{NH}_{\mathrm{x}} \text { e } \mathrm{NO}_{\mathrm{y}} \text { em Filoso } \\
\text { et al., (2006). A área total do campus foi obtida nos projetos de arquitetura fornecido pelo no setor de } \\
\text { planejamento da prefeitura do campus. }\end{array}$ \\
\hline $\begin{array}{l}\text { Res. das áreas } \\
\text { verdes }\end{array}$ & $\begin{array}{l}\text { A massa de resíduos das áreas verdes gerado foi obtida pelo setor administrativo do campus. A percentagem } \\
\text { média de nitrogênio na planta foi obtido a partir de Epstein (1965). }\end{array}$ \\
\hline Run off & $\begin{array}{l}\text { A deposição de nitrogênio atmosférico foi obtida a partir de mapas de deposição modelados de } \mathrm{NH}_{\mathrm{x}} \text { e } \mathrm{NO}_{\mathrm{y}} \text { de } \\
\text { filoso et al., (2006). A área urbana foi obtida nos projetos de arquitetura fornecido pelo setor de planejamento } \\
\text { do campus. }\end{array}$ \\
\hline
\end{tabular}

\section{Modelos Matemáticos}

Após obtenção dos dados do campus (Quadro 1), foi realizado o cálculo estimativa, adaptado do modelo de Savanick et al. (2007) para a realidade das universidades brasileiras. Para realizar toda estimativa foi necessário fazer uso das 18 equações a seguir:

Equação 1: Eletricidade

$N_{E m}=E N_{d e m} * C_{N O_{x} E_{m}} * 0,001 * 14 / 46$

Onde:

$N_{E m}=$ estimativa da emissão de $\mathrm{N}_{\mathrm{r}}$ da eletricidade em $\mathrm{kg}$

$E N_{\text {dem }}=$ energia demandada no ano $(\mathrm{kw})$

$C_{N O_{x} E_{m}}=$ fator de emissão de $\mathrm{NO}_{x}$ da energia $\left(\mathrm{g} \mathrm{NO}_{2} \mathrm{kwh}^{-1}\right)$

0,001 = fator de conversão de $\mathrm{g}$ em $\mathrm{kg}$

$14 / 46$ = conversão de $\mathrm{NO}_{2}$ em N

Equação 2: Estimativa da emissão de carros particulares

$E_{C C}=Q_{C C} * d *\left(C_{N O_{x} E_{m}} / 5\right) * m_{N / N O_{x}} * 0,001 * 200$

Onde:

$E_{C C}=$ estimativa da emissão de carros

$Q_{C C}=$ quant. média de carros que entram no campus $\times \mathrm{dia}^{-1}$ (und)

$d=$ distância média percorrida $(\mathrm{km})$ do carro $\times$ dia $^{-1}$

$C_{N O_{x} E_{m}}=$ fator de emissão de $\mathrm{NO}_{x}$ para carros $\left(\mathrm{g} \mathrm{N} \mathrm{km}^{-1}\right)$

$m_{N / N O_{x}}=$ proporção de $\mathrm{N}$ em NO${ }_{x}$

5 = lotação máxima do veículo

0,001 = fator de conversão $\mathrm{g}$ em $\mathrm{kg}$

200 = ano acadêmico (dias ativos)

Equação 3: Estimativa da emissão de ônibus

$E_{B C}=Q_{B C} * d *\left(\frac{B P_{N O_{x} E_{m}}}{46}\right) * m_{N / N O_{x}} * 0,001 * 200$

Onde:

$E_{B C}=$ estimativa da emissão de ônibus 
$Q_{B C}=$ pessoas que usam ônibus $x \mathrm{dia}^{-1}$

$d$ = distância percorrida $(\mathrm{km})$ pelo ônibus $\mathrm{x} \mathrm{dia}^{-1}$

$B P_{N O_{x} E_{m}}=$ fator de emissão de $\mathrm{NO}_{x}$ dos passageiros $\left(\mathrm{g} \mathrm{N} \mathrm{km}^{-1}\right)$

$m_{N / N O_{x}}=$ proporção de $\mathrm{N}$ em $\mathrm{NO}_{x}$

0,001 = fator de conversão de $\mathrm{g}$ em $\mathrm{kg}$

46 = lotação máxima do veículo

200 = ano acadêmico (dias ativos)

Equação 4: Estimativa da emissão de $\mathbf{N}$ dos carros oficiais

$E_{C O C}=\left(\left(d_{d} * D_{N O_{x} E_{m}}\right)+\left(d_{g} * G_{N O_{x} E_{m}}\right)\right) * m_{N / N O_{x}} * 0,001$

Onde:

$E_{C O C}=$ estimativa da emissão de $\mathrm{N}$ dos carros oficiais

$d_{d}=$ distância percorrida por carros movidos a diesel $(\mathrm{km})$

$D_{N O_{x} E_{m}}=$ fator de emissão de $\mathrm{NO}_{x}$ nos motores diesel $\left(\mathrm{g} \mathrm{N} \mathrm{km}^{-1}\right)$

$d_{g}=$ distância percorrida por carros movidos a gasolina $(\mathrm{km})$

$G_{N O_{x} E_{m}}=$ fator de emissão de $\mathrm{NO}_{x}$ nos motores gasolina $\left(\mathrm{g} \mathrm{N} \mathrm{km}^{-1}\right.$ )

$m_{N / N O_{x}}=$ proporção de $\mathrm{N} \mathrm{em} \mathrm{NO} \mathrm{N}_{x}$

0,001 = fator de conversão de $\mathrm{g}$ em $\mathrm{kg}$

Equação 5: Volume de combustível usado em voo

$V_{D T}=\left(F_{M C A 321} * d_{D T}\right) / A 321_{M R F T}$

Onde:

$V_{D T}=$ volume (I) de combustível usado em voo

$F_{M C A 321}=$ capacidade máx. do tanque da aeronave A321 (I)

$d_{D T}=$ distância percorrida nos voos demandados pela UFS $(\mathrm{km})$

$A 321_{\text {MRFT }}=$ distância máx. de voo de um A321com tanque cheio $(\mathrm{km})$

\section{Equação 6: Massa de combustível usado em voo}

$m_{D T}=v_{D T} * D_{A K}$

Onde:

$M_{D T}=$ massa ( $\mathrm{t}$ ) de combustível usado em voo

$V_{D T}=$ volume (I) combustível usado em voo

$D_{A K}=$ densidade do querosene de aviação (t/l)

Equação 7: Estimativa da emissão de $\mathbf{N}$ em voo

$N_{E_{m} D T}=\left(m_{D T} * A K_{E M} * m_{N / N O_{S}}\right) / 220$

Onde:

$N_{E_{m} D T}=$ estimativa da emissão de $\mathrm{N}$ em voo

$m_{D T}=$ massa ( $\mathrm{t}$ ) de combustível usada em voo

$A K_{E M}=$ fator de emissão de $\mathrm{NO}_{x}$ do querosene de aviação $\left(\mathrm{kg} \mathrm{N} \mathrm{t}^{-1}\right)$

$m_{N / N O_{S}}=$ Proporção de $\mathrm{N} \mathrm{em} \mathrm{NO}$ x

220 = capacidade de passageiros na aeronave $A 321$

\section{Equação 8: Estimativa da emissão de $\mathbf{N}$ no alimento humano}

$N_{I C Y}=m_{F I C} * \% N C B$

Onde:

$N_{I C Y}=$ estimativa de $\mathrm{N}$ contida nos alimentos do campus $\mathrm{x}$ ano-1

$m_{\text {FIC }}=$ massa $(\mathrm{kg})$ de alimentos que entram no campus $x$ ano-1 
$\%_{N C B}=$ Percentual de $\mathrm{N}$ de alimentos consumidos no campus

Equação 9: Estimativa da emissão de $\mathbf{N}$ no resíduo orgânico

$N_{D C}=m_{F D C} * \%_{N C B}$

Onde:

$N_{D C}=$ estimativa de $\mathrm{N}$ contido no resíduo orgânico $\times$ pessoa $^{-1} \times$ ano $^{-1}$

$m_{F D C}=$ massa $(\mathrm{kg})$ de alimento descartado (inclui o processo de preparação e desperdício de refeição)

$\%_{N C B}=$ percentual de $\mathrm{N}$ de alimentos consumidos no campus

Equação 10: Estimativa da emissão de $\mathbf{N}$ no efluente

$N_{S S}=N_{I C Y} * \%_{S S}$

Onde:

$N_{S S}=$ estimativa de $\mathrm{N}$ que segue para o esgoto

$N_{I C Y}=$ quantidade de $\mathrm{N}$ ingerida em nos alimentos do campus $\mathrm{x}$ ano-1

$\%_{S S}=$ percentual de $\mathrm{N}$ consumido no campus que vai para o esgoto

Equação 11: Estimativa da emissão de FBN no campus

$B N F_{C}=B N F_{B A R} * a_{G A C}$

Onde:

$B N F_{C}=$ estimativa da FBN no campus

$B N F_{B A R}=\mathrm{FBN}$ no ecossistema de Mata Atlântica

$a_{G A C}=$ área verde do campus (ha)

Equação 12: Estimativa da emissão da deposição atmosférica de $\mathrm{N}$ na área de estudo

$N_{A D}=\left(m_{N O y h a} * m_{\frac{N}{N O y}}\right)+\left(m_{N H x h a} * m_{\frac{N}{N H x}}\right)$

Onde:

$N_{A D}=$ deposição atmosférica de $\mathrm{N}$ para área de estudo

$m_{N O y h a}=$ massa de $\mathrm{NO}_{\mathrm{y}}$ depositada por hectare na área de estudo

$m_{\frac{N}{N O y}}=$ proporção de $\mathrm{N} \mathrm{em} \mathrm{NO}_{y}$

$m_{N H x h a}=$ massa de $\mathrm{NH}_{\mathrm{x}}$ depositada por hectare na área de estudo

$m_{\frac{N}{N H x}}=$ proporção de $\mathrm{N}$ em $\mathrm{NH}_{\mathrm{x}}$

Equação 13: Estimativa da emissão da deposição atmosférica total de $\mathbf{N}$ no campus

$N_{T A D}=N_{A D} * a_{T A C}$

Onde:

$N_{T A D}=$ deposição atmosférica de $\mathrm{N}$ total no campus

$N_{A D}=$ deposição atmosférica de $\mathrm{N}$ para área de estudo

$a_{T A C}=$ área total do campus (ha)

Equação 14: Estimativa da emissão de $\mathbf{N}$ nos resíduos de jardinagem

$N_{G W}=V_{G W} * D_{G W} * 12 * \%_{N P}$

Onde:

$N_{G W}=$ estimativa de $\mathrm{N}$ em resíduos de jardinagem

$V_{G W}=$ volume de resíduos de jardinagem coletados a cada mês no campus

$D_{G W}=$ densidade dos resíduos de jardinagem 
$12=$ meses do ano

$\%_{N P}=$ percentagem de $\mathrm{N}$ em plantas

\section{Equação 15: Estimativa da emissão de $\mathbf{N}$ drenado via runoff}

$N_{R O}=N_{A D} * a_{U A C}$

Onde:

$N_{R O}=$ estimativa de $\mathrm{N}$ drenado no runoff

$N_{A D}=$ deposição atmosférica de $\mathrm{N}$ para área de estudo

$a_{U A C}=$ área urbana do campus (ha)

\section{RESULTADOS}

A partir dos dados fornecidos pelo setor administrativo do Campus São Cristóvão da UFS e pelos fatores de conversão de nitrogênio relacionados a produção de eletricidade, combustíveis tomados a partir do "Inventário nacional de emissões atmosféricas por veículos automotores rodoviários" (MMA, 2013), alimentos, efluentes e fixação de nitrogênio foi possível aplicar os modelos apresentados propiciando o cálculo do nitrogênio reativo emitido pelas atividades elencadas nesse estudo (Quadro 2).

Quadro 2: Cálculo das emissões de Nitrogênio no Campus São Cristóvão da UFS entre 2015 a 2016.

\begin{tabular}{|c|c|c|c|}
\hline Fontes de nitrogênio & Dados fornecidos & $\begin{array}{l}\text { Equação utilizada e fator } \\
\text { aplicado }\end{array}$ & $\begin{array}{l}\text { Valor calculado de } \mathbf{N}-\mathrm{kg} \\
\mathrm{N} \text { ano } \\
\end{array}$ \\
\hline Eletricidade & $23.007 .066 \mathrm{kWh}$ & $\begin{array}{l}\text { Equação } 01 \\
0,21 \mathrm{~g} \mathrm{NO}_{2} / \mathrm{kWh}\end{array}$ & $1.470,45$ \\
\hline \multicolumn{4}{|l|}{ Transportes terrestres } \\
\hline -Veículos leves a álcool e gasolina & $\begin{array}{l}6.201 \text { pessoas } \\
22,2 \mathrm{~km} \mathrm{dia}^{-1}\end{array}$ & $\begin{array}{l}\text { Equação } 02 \\
0,03 \mathrm{gNO}_{2} \mathrm{~km}^{-1}\end{array}$ & 24,63 \\
\hline -Veículos leves a diesel & $\begin{array}{l}76 \text { pessoas } \\
22,2 \mathrm{~km} \mathrm{dia}^{-1}\end{array}$ & $\begin{array}{l}\text { Equação } 02 \\
0,06 \mathrm{gNO}_{2} \mathrm{~km}^{-1}\end{array}$ & 0,60 \\
\hline -Vans movidas a diesel & $\begin{array}{l}470 \text { pessoas } \\
37,4 \mathrm{~km} \mathrm{dia}^{-1}\end{array}$ & $\begin{array}{l}\text { Equação } 02 \\
1,15 \mathrm{gNO}_{2} \mathrm{~km}^{-1}\end{array}$ & 50,24 \\
\hline -Ônibus a diesel & $\begin{array}{l}10.556 \text { pessoas } \\
45,8 \mathrm{~km} \mathrm{dia}^{-1}\end{array}$ & $\begin{array}{l}\text { Equação } 03 \\
1,29 \mathrm{gNO}_{2} \mathrm{~km}^{-1}\end{array}$ & 404,38 \\
\hline -Carros oficiais & $\begin{array}{l}1.714 \mathrm{~km} \mathrm{dia}^{-1} \text { (gasolina) } \\
2.350 \mathrm{~km} \mathrm{dia}^{-1} \text { (diesel) }\end{array}$ & $\begin{array}{l}\text { Equação } 04 \\
0,03 \mathrm{gNO}_{2} \mathrm{~km}^{-1} \\
1,15 \mathrm{gNO}_{2} \mathrm{~km}^{-1} \\
\end{array}$ & 81,35 \\
\hline Transporte aéreo & $565.100 \mathrm{~km}$ percorridos & $\begin{array}{l}\text { Equações 05, } 06 \text { e } 07 \\
14,1 \mathrm{~kg} \mathrm{~N} \mathrm{t}^{-1}\end{array}$ & $10.354,61$ \\
\hline Alimentos que entram no Campus & $\begin{array}{l}1.059 .960 \mathrm{~kg} \text { de alimentos por } \\
\text { ano }\end{array}$ & $\begin{array}{l}\text { Equação } 08 \\
1,34 \% \text { de cada alimento é } \mathrm{N}\end{array}$ & $14.203,46$ \\
\hline Resíduos dos alimentos & $111.560 \mathrm{~kg} \mathrm{ano}^{-1}$ & $\begin{array}{l}\text { Equação } 09 \\
1,34 \% \text { do alimento total } \\
\text { adquirido é } \mathrm{N}\end{array}$ & $1.494,90$ \\
\hline $\begin{array}{l}\text { Efluentes gerados pelo consumo de } \\
\text { alimentos }\end{array}$ & $948.400 \mathrm{~kg}^{2} \mathrm{ano}^{-1}$ & $\begin{array}{l}\text { Equação } 10 \\
1,34 \% \text { do alimento total } \\
\text { adquirido é } \mathrm{N}\end{array}$ & $11.437,70$ \\
\hline FBN & 109,9 ha de mata atlântica & $\begin{array}{l}\text { Equação } 11 \\
21,6 \mathrm{~kg} \mathrm{~N} \mathrm{ha}^{-1} \text { ano-1 }^{-1}\end{array}$ & $2.373,84$ \\
\hline Deposição atmosférica & 154,3 ha área no campus & $\begin{array}{l}\text { Equação } 13 \\
1,59 \mathrm{~kg} \mathrm{~N} \mathrm{ha}^{-1} \text { ano }^{-1}\end{array}$ & 245,33 \\
\hline Resíduos de áreas verdes & $\begin{array}{l}105.000 \mathrm{~kg} \text { ano-1 de resíduos } \\
\text { de podas }\end{array}$ & $\begin{array}{l}\text { Equação } 14 \\
1,59 \mathrm{~kg} \mathrm{~N} \mathrm{ha}^{-1} \mathrm{ano}^{-1}\end{array}$ & $1.575,00$ \\
\hline Run off de N & 44,4 ha de área urbana & $\begin{array}{l}\text { Equação } 15 \\
1,59 \mathrm{~kg} \mathrm{~N} \mathrm{ha}^{-1} \mathrm{ano}^{-1}\end{array}$ & 70,60 \\
\hline \multicolumn{3}{|l|}{ TOTAL } & $43.787,09$ \\
\hline
\end{tabular}




\section{DISCUSSÃO}

Pelos valores de emissões obtidos, pode-se observar que três atividades estão acima de $10.000 \mathrm{~kg}$ N.ano ${ }^{-1}$, contribuindo de forma relevante no valor total das emissões geradas $(82,21 \%)$, essas áreas estão relacionadas aos alimentos (consumo), efluentes domésticos gerados e uso de transporte aéreo. $\mathrm{O}$ segundo grupo de atividades com maior contribuição $(15,79 \%)$ nas emissões são a Fixação Biológica de Nitrogênio (FBN), resíduos de áreas verdes e de alimentos e energia elétrica consumida. Com $2 \%$ de impacto no total de emissões geradas estão as atividades relacionadas a transporte terrestre (ônibus, vans e carros), além da deposição atmosférica de nitrogênio e do escoamento superficial nas áreas urbanizadas do campus. Esse comportamento pode ser melhor identificado na Figura 1.

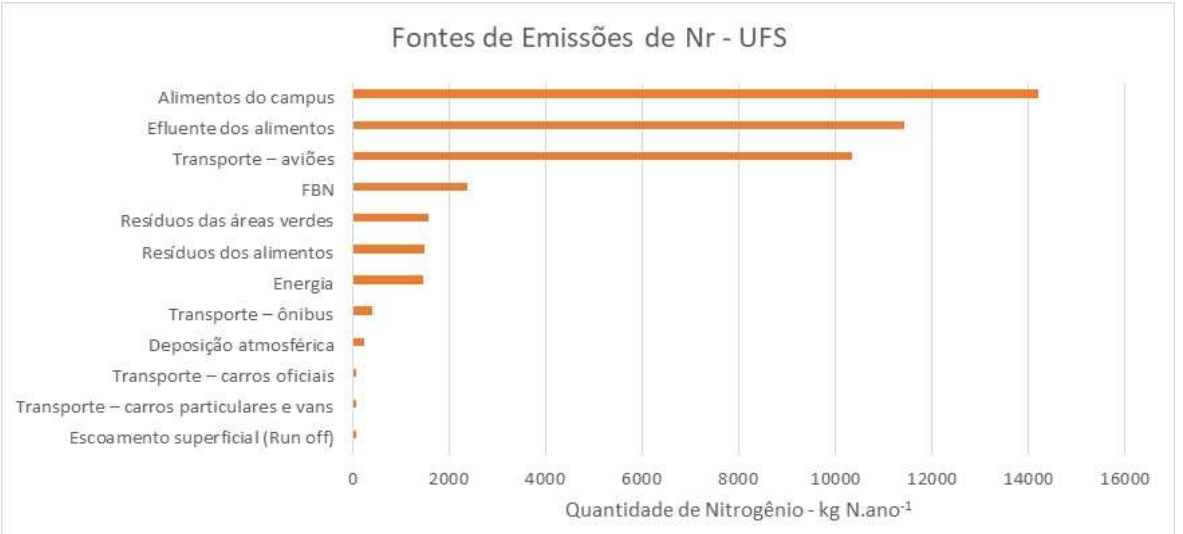

Figura 1: Síntese geral das emissões de Nitrogênio no Campus São Cristóvão da UFS.

A partir do cenário traçado em função das três maiores emissões de nitrogênio contabilizadas e tendo em vista a lógica da máxima promoção da sustentabilidade referida a IES, sugere-se a este campus, algumas alternativas voltadas a redução dessas emissões, tendo em vista as ações preventivas pautadas em conceitos e métodos de tecnologias limpas e ecologia industrial tais como: Reduzir no que for possível as viagens aéreas, incentivando o uso de ônibus para viagens a nível nacional, bem como incentivar o uso de tecnologias virtuais em casos de avaliação de trabalhos; Promover estudos que viabilizem a utilização da urina humana gerada do campus possibilitando formas de segregação, tratamento, armazenamento e aplicação, a fim de usar seu nitrogênio como fertilizante nas áreas verdes do campus, ou fornecê-la como um insumo agrícola fora do campus.

Por outro lado, mesmo entendendo uma representatividade menor no tocante às emissões de $\mathrm{N}$, seguem algumas alternativas de ações voltadas para à redução das emissões no referido campus: A substituição de parte da eletricidade usada no campus por outra fonte de energia mais limpa (como a energia solar ou eólica), mediante estudo de viabilidade; a melhoria das estruturas de edifícios, a fim de promover uma maior ventilação e iluminação natural evitando o uso de equipamentos consumidores de energia (ar condicionado, lâmpadas etc.); a aquisição de equipamentos e acessórios que consomem menos energia; Incentivar o uso de transporte menos impactante a exemplo do uso de bicicletas, inclusive dentro e fora da comunidade acadêmica (quando for possível, pois é evidente tanto o problema da mobilidade urbana como a falta de segurança pública nas cidades) com o objetivo de reduzir significativamente as emissões de $\mathrm{N}_{\mathrm{r}}$ de 
transporte; No paisagismo campus, priorizar a introdução de espécies nativas. Em alguns casos, quando se busca a recuperação de solos degradados, é importante introduzir espécies de crescimento precoce, pois, capturam mais carbono e nitrogênio, a fim de promover mais a fertilidade do solo; Reduzir o descarte de resíduos de alimentos através de métodos de compostagem; Reduzir o descarte de resíduos de jardim através de métodos de compostagem; Evitar a devastação área verde para preservar a biodiversidade e preservar a estrutura do solo e seus processos associados;

Um exemplo positivo para a sustentabilidade da UFS é a criação da UFS ambiental, institucionalizado fevereiro de 2012 e que tem por objetivo seguir as diretrizes propostas pela norma ISO 14000, buscando evidenciar ações e comportamentos ambientais para Universidade, contribuindo assim para a sustentabilidade ambiental e promovendo práticas ambientais em todos os setores da UFS. Além disso, outras ações estão sendo direcionadas às seguintes questões: gestão de resíduos, licitação sustentável, qualidade de vida no ambiente de trabalho, sensibilização de capacitação dos servidores, uso racional de recursos e construções sustentáveis.

\section{CONCLUSÕES}

As atividades humanas já convertem mais nitrogênio da atmosfera em formas reativas do que todos os processos naturais da Terra em conjunto. Por isso, o nitrogênio tem sido uma das principais causas da perda de biodiversidade planetária, seus efeitos na atmosfera afetam qualidade do ar causando poluição atmosférica, partículas em suspensão, ozônio troposférico, e como consequência direta afeta a saúde humana, a qualidade da água doce por conta dos nitratos, eutrofização dos mananciais além de zonas mortas e destruição do ozônio estratosférico.

A estimativa das emissões de Nitrogênio no Campus central da UFS, localizado na cidade de São Cristóvão/SE, resultou em um valor de 43.787,09 $\mathrm{kg}$ de N ano-1 (aproximadamente 43,8 toneladas de N.ano$\left.{ }^{1}\right)$, tomando como base os anos letivos de 2015 e 2016. Através deste estudo, foi possível analisar muitos aspectos ambientais intrinsecamente ligadas às atividades do campus, tais como o consumo de energia, transporte, práticas de jardinagem, consumo de alimentos, geração de efluentes e outros aspectos responsáveis por diversos impactos ambientais no campus.

O caminho para a sustentabilidade do campus exige numerosos esforços que vão além da avaliação do fluxo de $\mathrm{N}$ no campus, tais como avaliação do carbono, uso racional de recursos naturais, adoção de estratégias mais sustentáveis do ponto de vista da energia e transporte dentre outras que a UFS ambiental poderia pleitear. A fim de implementar práticas mais sustentáveis nas universidades, sugere-se adoção do uso de tecnologias limpas (métodos preventivos) para reduzir a intensidade de cada fluxo de $\mathrm{N}_{\mathrm{r}}$ e até mesmo de carbono no campus. Além disso, cabe ressaltar que a mitigação dos impactos ambientais depende também de outras ações a exemplo da regulamentação de leis e mecanismos de controle.

\section{REFERÊNCIAS}

ANAC. Inventário nacional de emissões atmosféricas da aviação civil. Agência Nacional de Aviação Civil, 2014. 
ERISMAN, J. W.; BLEEKER, A.; GALLOWAY, J.; SUTTON, M. A.. Reduced nitrogen in ecology and the environment. Environmental Pollution, v.150, n.1, p.140-149, 2007. DOI: http://doi.org/10.1016/j.envpol.2007.06.033

ERISMAN, J. W.; GALLOWAY, J. N.; SUTTON, M. A.; KLIMONT, Z.; WINIWATER, W.. How a century of ammonia synthesis changed the world. Nature Geoscience, v.1, p.636-639, 2008. DOI: http://doi.org/10.1038/ngeo325

ERISMAN, J. W.; GALLOWAY, J. N.; SEITZINGER, S.; BLEEKER, A.; BUTTERBACH-BAHL, K.. Reactive nitrogen in the environment and its effect on climate change, Current Opinion. Environmental Sustainability, v.3, n.5, p.281-290, 2011. DOI: http://doi.org/10.1016/j.cosust.2011.08.012

ERISMAN, J. W.; GALLOWAY, J. N.; SEITZINGER, S.; BLEEKER, A.; DISE, N. B.; PETRESCU, A. M. R.; LEACH, A. M.; VRIES, W.. Consequences of human modification of the global nitrogen cycle. Philos. T. Roy. Soc. B., v.368, 2013. DOI: http://doi.org/10.1098/rstb.2013.0116

ERISMAN, J. W.; GALLOWAY, J. N.; DICE, N. B.; SUTTON, M. A.; BLEEKER, A.; GRIZZETTI, B.; LEACH, A. M.; VRIES, W. Nitrogen: too much of a vital resource. Science Brief. Zeist: WWF, 2015.

EPSTEIN, E.. Plant Biochemistry. New York: Academic, 1965.

FILOSO, S.; MARTINELLI, L. A.; HOWARTH, R. W.; BOYER, E. W.; DENTENER, F.. Human activities changing the nitrogen cycle in Brazil, Biogeochemistry, n.1-2, p.61-89, 2006. DOI: http://doi.org/10.1007/s10533-006-9003-0

FOWLER, D.; COYLE, M.; SKIBA, U.; SUTTON, M. A.; CAPE, J. N.; REIS, S.; SHEPPARD, L. J.; JENKINS, A.; GRIZZETTI, B.; GALLOWAY, J. N.; VITOUSEK, P.; LEACH, A., BOUWMAN, A. F.; BUTTERBACH-BAHL, K.; DENTENER, F.; STEVENSON, D.; AMANN, M.; VOSS, M.. The global nitrogen cycle in the twenty-first century, Philos. T. Roy. Soc. B., v.368, 2013. DOI: http://doi.org/10.1098/rstb.2013.0165

FOWLER, D.; STEADMAN, C. E.; STEVENSON, D.; COYLE, M.; REES, R. M.; SKIBA, U. M.; SUTTON, M. A.; CAPE, J. N.; DORE, A. J.; VIENO, M.; SIMPSON, D.; ZAEHLE, S.; STOCKER, B. D.; RINALDI, M.; FACCHINI, M. C.; FLECHARD, C. R.; NEMITZ, E.; TWIGG, M.; ERISMAN, J.W.; GALLOWAY, J. N.. Effects of global change during the 21st century on the nitrogen cycle. Atmospheric Chemistry and Physics Discuss., v.15, p.17471868, 2015. DOI: 10.5194/acp-15-13849-2015

GALLOWAY, J.N. The global nitrogen cycle: Changes and consequences. Environmental Pollution, v.102, n.1, p.15-24, 1998. DOI: http://doi.org/10.1016/S0269-7491(98)80010-9

GALLOWAY, J. N.. The Global Nitrogen Cycle. Treatise on Geochemistry, v.8, p.557-583, 2003.

GALLOWAY, J. N.; ABER, J. D.; ERISMAN, J. W.; SEITZINGER, S. P.; HOWARTH, R. W.; COWLING, E. B.; COSBY, B. J.. The Nitrogen Cascade. BioScience, v.53, n.4, p.341-356, 2003. DOI: http://doi.org/10.1641/00063568(2003)053[0341:TNC]2.0.CO;2

GALLOWAY, J. N.; DENTENER, F. J.; CAPONE, D. G.; BOYER, E. W.; HOWARTH, R. W.; SEITZINGER, S. P.; ASNER, G. P.; CLEVELAND, C. C.; GREEN, P. A.; HOLLAND, E. A.; KARL, D. M.; MICHAELS, A. F.; PORTER, J. H.; TOWNSEND, A. R.;
VOROSMARTY, C. J.. Nitrogen cycles: past, present and future. Biogeochemistry, v.70, n.2, p.153-226, 2004. DOI: http://doi.org/10.1007/s10533-004-0370-0

GALLOWAY, J. N.; TOWNSEND, A. R.; ERISMAN, J. W.; BEKUNDA, M.; CAI, Z.; FRENEY, J. R.; MARTINELLI, L. A.; SEITZINGER, S. P.; SUTTON, M. A.. Transformation of the Nitrogen Cycle: Recent Trends, Questions, and Potential Solutions. Science, v.320, n.5878, p.889-892, 2008. DOI: http://doi.org/10.1126/science.1136674

GALLOWAY, J. N.; LEACH, A. M.; BLEEKER, A.; ERISMAN, J. W.. A chronology of human understanding of the nitrogen cycle. Philosophical Transactions of the Royal Society B., v.368, p.01-20, 2013. DOI: http://doi.org/10.1098/rstb.2013.0120

GALLOWAY, J. N; WINIWARTER, W.; LEIP, A.; LEACH, A. M.; BLEEKER, A.; ERISMAN, J. W.. Nitrogen footprints: past, present and future. Environmental. Research., v.9, n.11, 2014. DOI: http://doi.org/10.1088/1748-9326/9/11/115003

LEACH, A. M.; MAJIDI, A. N.; GALLOWAY, J. N.; GREENE, A. J.. Toward Institutional Sustainability: A Nitrogen Footprint Model for a University. Research and Solutions. v.6, n.4 2013. DOI: http://doi.org/10.1089/sus.2013.9852

MMA. Ministério do Meio Ambiente. Inventário nacional de emissões atmosféricas por veículos automotores rodoviários. 2013.

ROCKSTROM, J.; STEFFEN, W.; NOONE, K.; PERSSON, A.; CHAPIN, F. S.; LAMBIN, E.; LENTON, T. M.; SCHEFFER, M.; FOLKE, C.; SCHELLNHUBER, H.; NYKVIST, B.; DE WIT, C. A.; HUGHES, T.; VAN DER LEEUW, S.; RODHE, H.; SORLIN, S.; SNYDER, P. K.; COSTANZA, R.; SVEDIN, U.; FALKENMARK, M.; KARLBERG, L.; CORELL, R. W.; FABRY, V. J.; HANSEN, J.; WALKER, B.; LIVERMAN, D.; RICHARDSON, K.; CRUTZEN, P.; FOLEY, J.. Planetary Boundaries: Exploring the safe operating space for humanity. Ecology and society Journal, v.14, n.2, p.32, 2009. DOI: http://doi.org/10.5751/ES-03180-140232

SAVANICK, S., BAKER L., PERRY., J. Case study for evaluating campus sustainability: nitrogen balance for the University of Minnesota. Urban Ecosystems, v.10, n.2, p.119-137, 2007. DOI: http://doi.org/10.1007/s11252-007-0018-7

SMIL, V. Nitrogen and food production: proteins for human diets. Ambio, v.31, n.2, p.126-131, 2002. DOI: http://doi.org/10.1579/0044-7447-31.2.126

STEFFEN, W.; RICHARDSON, K.; ROCKSTRÖM, J.; CORNELL, S.E.; FETZER, I.; BENNETT, E. M.; BIGGS, R.; CARPENTER, S. R.; VRIES, W.; WIT, C. A.; FOLKE, C.; GERTEN, D.; HEINKE, J.; MACE, G. M.; PERSSON, L. M.; RAMANATHAN, V.; REYERS, B.; SÖRLIN, S.. Planetary boundaries: Guiding human development on a changing planet. Science, v.347, n.6223, 2015. DOI: http://doi.org/10.1126/science.1259855

UMACS. Sustainability organizations in higher education. Upper midwest association for campus sustainability. 2013.

UNEP. Emerging issues update: Excess Nitrogen in the Environment. Year Book, 2014.

UNICAMP. Brazilian Table Food Composition. University of Campinas, 2011.

A CBPC - Companhia Brasileira de Produção Científica (CNPJ: 11.221.422/0001-03) detém os direitos materiais desta publicação. Os direitos referem-se à publicação do trabalho em qualquer parte do mundo, incluindo os direitos às renovações, expansões e disseminações da contribuição, bem como outros direitos subsidiários. Todos os trabalhos publicados eletronicamente poderão posteriormente ser publicados em coletâneas impressas sob coordenação da Sustenere Publishing, da Companhia Brasileira de Produção Científica e seus parceiros autorizados. Os (as) autores (as) preservam os direitos autorais, mas não têm permissão para a publicação da contribuição em outro meio, impresso ou digital, em português ou em tradução. 